\title{
Geometric potential and transport in photonic topological crystals
}

\author{
Alexander Szameit ${ }^{1 *}$, Felix Dreisow ${ }^{2}$, Matthias Heinrich ${ }^{2}$, Robert \\ Keil $^{2}$, Stefan Nolte ${ }^{2}$, Andreas Tünnermann ${ }^{2}$, Stefano Longhi ${ }^{3}$ \\ ${ }^{1}$ Physics Department and Solid State Institute, Technion, 32000 Haifa, Israel \\ ${ }^{2}$ Institute of Applied Physics, Friedrich-Schiller-Universität, \\ Max-Wien-Platz 1, 07743 Jena, Germany and \\ ${ }^{3}$ Dipartimento di Fisica, Politecnico di Milano, \\ Piazza Leonardo da Vinci 32, 20133 Milan, Italy
}

\begin{abstract}
We report on the experimental realization of an optical analogue of a quantum geometric potential for light wave packets constrained on thin dielectric guiding layers fabricated in silica by the femtosecond laser writing technology. We further demonstrate the optical version of a topological crystal, with the observation of Bloch oscillations and Zener tunneling of purely geometric nature.
\end{abstract}

PACS numbers: 03.65.Ge, 42.82.Et, 78.67.Pt 
Studying the quantum mechanics of a particle constrained on a curved space [1 3 ] has been a matter of great controversies for more than 50 years. For a long time, it has been known that geometry influences in different ways the motion of classical and quantum particles confined on a curved surface or on a line. When surface curvatures become comparable to the de Broglie wavelength, geometrical effects come into play by the internal metric of the constraint manifold as well as by the external metric of the embedding space. As opposed to a classical particle, a quantum particle retains some knowledge of the surrounding threedimensional space and, in spite of the absence of interactions, it experiences an effective frictional potential of geometric nature [2, 3]. Such a geometric potential is of major importance in the understanding and control of the physical properties of novel low-dimensional functional materials, like curved carbon nanotubes and DNA wires [4]. For instance, an electron confined to a periodically curved surface senses a periodic frictional potential which acts as a topological crystal [5]. The definition of a geometric potential in quantum and condensed matter physics, however, is troubled by the realization of a proper squeezing procedure [6, 7], which is needed to avoid operator-ordering ambiguities [8, 9]. A rather accepted approach that avoids operator-ordering ambiguities is the confining-potential approach, originally proposed by Jensen, Koppe and da Costa (JKC) [2, 3], in which a strong force acting normal to the surface provides the appropriate confinement. Quantum excitation energies in the normal direction are raised far beyond those in the tangential direction. Hence, the particle motion normal to the surface can be safely neglected, which leads to an effective Hamiltonian for propagation along the curved surface. Though the JKC approach has gained a broad consensus especially in the theoretical condensed matter physics community [10 14], it was noticed that such an ideal squeezing procedure may correspond to unrealistic restrictions or cannot be unique [6, 7]. Clear evidences of geometric potentials in low-dimensional curved nanosystems are still lacking and are likely to be a controversial matter. On the other hand, optics has offered in recent years a fascinating laboratory tool to investigate classical analogues of otherwise inaccessible quantum-mechanical and relativistic effects (see, e.g., [15-19]). In this Letter we report on the first experimental realization of an optical analogue of a quantum geometric potential for light wave packets constrained on thin dielectric guiding layers fabricated in silica by the femtosecond laser writing technology [20]. We further demonstrate the optical version of a topological crystal [5], with the observation of Bloch oscillations and Zener tunneling of purely geometric nature [21]. 
Let us consider wave propagation at wavelength $\lambda$ in a thin and weakly guiding dielectric layer, which is assumed to be invariant along the $z$ direction and arbitrarily curved in the transverse $(x, y)$ plane along a curve $\gamma$ (see Fig.1a). In the scalar approximation, the electric field $E(x, y, z)$ satisfies the Helmholtz equation

$$
\nabla_{t}^{2} E+\partial_{z}^{2} E+(n / \lambda)^{2} E=0
$$

where $\nabla_{t}^{2}=\partial_{x}^{2}+\partial_{y}^{2}$ is the transverse Laplacian, $\lambda=\lambda /(2 \pi)$ is the reduced wavelength, and $n=n(x, y)$ is the refractive index profile of the optical structure, which is assumed to be independent of $z$ and weakly deviating from the refractive index $n_{s}$ of the substrate. The analogy between spatial propagation of light waves along the $z$ direction and the temporal dynamics of a two-dimensional quantum particle constrained on the curved line $\gamma$, discussed by da Costa [3], is at best captured by the introduction of the local curvilinear coordinates $(\sigma, \eta)$ of Fig.1a. The volume element in the curvilinear system is given by $d V=\chi d \sigma d \eta d z$, where $\chi(\sigma, \eta)=1+\eta / R$ and $R=R(\sigma)$ is the local radius of curvature of $\gamma$. To study the behavior of light waves near the guiding layer $(\eta \rightarrow 0)$, following [3] it is worth introducing the new wave field $\mathcal{E}=E \sqrt{\chi}$. After writing in Eq.(1) the transverse Laplacian $\nabla_{t}^{2}$ in curvilinear coordinates and with the substitution $E=\mathcal{E} / \sqrt{\chi}$, the evolution equation of the field $\mathcal{E}$ in the limit $\eta \rightarrow 0$ (i.e., close to the line $\gamma$ ) reads

$$
\left(\partial_{\sigma}^{2}+\partial_{\eta}^{2}+\partial_{z}^{2}\right) \mathcal{E}+\left(1 / 4 R^{2}\right) \mathcal{E}+(n / \lambda)^{2} \mathcal{E}=0
$$

In the weak guiding limit $\left|n(\sigma, \eta)-n_{s}\right| \ll n_{s}$ and assuming $|R| \gg \lambda$, the paraxial approximation can be introduced in the usual way by setting $\mathcal{E}(\sigma, \eta, z)=\psi(\sigma, \eta, z) \exp \left(i n_{s} z / \lambda\right)$ and neglecting $\partial_{z}^{2} \psi$ as compared to $(1 / \lambda) \partial_{z} \psi$. This yields the optical Schrödinger equation

$$
i \lambda \frac{\partial \psi}{\partial z}=-\frac{\lambda^{2}}{2 n_{s}}\left(\frac{\partial^{2}}{\partial \sigma^{2}}+\frac{\partial^{2}}{\partial \eta^{2}}\right) \psi+\left[V_{c}(\sigma, \eta)+V_{g}(\sigma)\right] \psi
$$

where $V_{c}(\sigma, \eta)=\left[n_{s}^{2}-n^{2}(\sigma, \eta)\right] /\left(2 n_{s}\right) \simeq n_{s}-n(\sigma, \eta)$ is the confining potential and

$$
V_{g}(\sigma)=-\frac{\lambda^{2}}{8 n_{s} R^{2}(\sigma)}
$$

is the so-called geometric potential [3]. The correspondence of Eq.(3) to the quantummechanical Schrödinger equation of a two-dimensional particle constrained on the curve $\gamma$, discussed by da Costa [3], is formally obtained after replacing the photon wavelength $\lambda$ with the Planck constant $h$, the refractive index $n_{s}$ with the particle mass $m$ and the spatial 
coordinate $z$ with time $t$. Hence, in optics the quantum-mechanical evolution in time of a two-dimensional wave function is mapped onto the propagation of an optical wave packet along the spatial $z$-direction. The $z$-independence of the path $\gamma$ reflects the circumstance that the constraint is time-independent. Similarly to the quantum mechanical problem [3], in the optical Schrödinger equation (3) the confining potential $V_{c}$, squeezing the wave packet around $\gamma$, originates from the physical change of the refractive index in the guiding layer (see Fig.1b); on the contrary, the geometric potential $V_{g}$ is of geometric nature and arises from the diffraction operator $-\left(\lambda^{2} / 2 n_{s}\right) \nabla_{t}^{2}$ (the analogue of the kinetic energy operator) in curvilinear coordinates. In the curved reference frame $(\sigma, \eta)$, this potential acts on light waves like a fictitious refractive index change.

In the JKC approach, the confining potential $V_{c}$ is taken to be independent of $\sigma$, a condition which ensures that the constraint is frictionless in the classical (geometric-optic) limit. In this case, the motion in the normal $\eta$ direction can be exactly separated from the dynamics by letting $\psi(\sigma, \eta, z)=F(\sigma, z) g(\eta) \exp \left(-i E_{0} z / \lambda\right)$, where $g(\eta)$ is the ground-state wave function of the confining potential $V_{c}$ and $E_{0}$ its corresponding energy [i.e., $-\left(\lambda^{2} / 2 n_{s}\right)\left(d^{2} g / d \eta^{2}\right)+$ $\left.V_{c}(\eta) g(\eta)=E_{0} g(\eta)\right]$. One then obtaines an effective equation for the particle motion along the $\sigma$ coordinate

$$
i \star \frac{\partial F}{\partial z}=-\frac{\lambda^{2}}{2 n_{s}} \frac{\partial^{2} F}{\partial \sigma^{2}}+V_{g}(\sigma) F .
$$

Therefore, the geometric potential $V_{g}$ acts as an effective frictional potential for the motion on the curved manifold. Unfortunately, there is no compelling reason that actual confining potentials have this exceptional property [6]. Moreover, it was shown that one can construct ad-hoc confining potentials leading to an arbitrary correction to the geometric potential that obey the frictionless condition of the constraint in the classical limit $\lambda \rightarrow 0$ [7]. Nonideal squeezing potentials will generally replace the geometric potential $V_{g}$ in Eq.(5) by an effective frictional potential $V_{e f f}$. If we allow the confining potential $V_{c}$ to slowly vary with $\sigma$, the tangential motion can still be approximately separated from the normal one by using a multiple scale asymptotic analysis (as in [7]). In this way, the tangential motion turns out to be governed again by the reduced Schrödinger equation (5), but with the geometric potential $V_{g}$ replaced by $V_{e f f}=V_{g}+\Delta V_{g}$, where

$$
\Delta V_{g}(\sigma)=\Delta E(\sigma)-\frac{\lambda^{2}}{2 n_{s}} \int d \eta g \frac{\partial^{2} g}{\partial \sigma^{2}} .
$$

In the above equation, $g(\sigma, \eta)$ is the local ground-state wave function of the confining poten- 
tial $V_{c}$, normalized such that $\int d \eta g^{2}=1$, and $E_{0}+\Delta E(\sigma)$ is the corresponding $\sigma$-dependent energy, i.e. $-\left(\lambda^{2} / 2 n_{s}\right) \partial_{\eta}^{2} g+V_{c} g=\left(E_{0}+\Delta E\right) g$. The JKC geometric potential is attained whenever $\Delta V_{g}(\sigma)$ vanishes, a condition which does not necessarily imply the $\sigma$-invariance of $V_{c}$.

In order to demonstrate the features of an ideal JKC squeezing procedure and the discrepancies of a non-ideal squeezing, we realized an optical analog of a topological crystal [5], in which a two-dimensional wave function is squeezed onto an undulating curve $\gamma$. To this aim, a sinusoidally undulated slab waveguide (Fig. 1k), defined by $y(x)=A \sin (2 \pi x / \Lambda)$, was fabricated using the laser direct-writing technology [20]. The use of this technique commonly yields a layer which exhibits constant thickness in the vertical $y$-direction rather then in the normal $\eta$-direction. For a homogeneous refractive index change as shown in Fig. $1 \mathrm{~d}$ d, this implies that the correction to the geometric potential does not vanish, i.e. $\Delta V_{g} \neq 0$. However, using the laser direct-writing approach, the refractive index variations along $\gamma$ can be tailored with great accuracy. In particular, it is possible to modulate the strength of $n(\sigma, \eta)$ along $\sigma$ such that $\Delta V_{g}$ gets negligible as compared to $V_{g}$. The refractive index distribution that closely approximates the JKC condition $\Delta V_{g}=0$ is shown in Fig. 1k, and corresponds to the highest index change at the inflection points of the undulation, where $R=\infty$.

To investigate wave packet dynamics in the topological crystal, we employ a fluorescence microscopy technique [22]. Light around $\lambda=633 \mathrm{~nm}$ excites color centers, which are formed during the waveguide fabrication process. The resulting fluorescence is proportional to the intensity of the propagating light, and can be observed from above the sample, as sketched in Fig. 2, due to the isotropic emission. When launching a beam into the two samples with the refractive index profiles representing the non-ideal (Fig. 1 $1 \mathrm{~d}$ ) and the ideal (JKC) case (Fig. 15), one obtains the intensity distributions shown in Fig. Ba,c, respectively. The observations are confirmed by numerical simulations of the full wave equation (see Fig. 3b,d). Although in both cases the initial wave packet spreads, the spreading rate is larger for the JKC potential. Most importantly, the spreading pattern observed in Fig.3c reproduces with excellent accuracy the diffraction pattern in a one-dimensional crystal with the potential defined by Eq.(4). Hence, our experimental technique enables to squeeze a wave packet mimicking the ideal JKC method. It is important to point out that the equivalent lattice potential $V_{g}$ caused by the curvature of the JKC guide has a geometric origin and cannot be 
naively explained by effective-index or variational methods generally adopted in guided-wave optics [23]. As the effective index method well explains the formation of periodic optical potentials in common waveguide array settings (such as those investigated in [24]), it fails to explain the onset of the geometric potential for the JKC undulating slab structure of Fig.1e. In fact, if one calculates from Eq.(1) the effective index along the vertical $y$ direction using a separation variable method [23], the resulting effective index $n_{e}(x)$ [25] turns out to be largest in the regions with vanishing curvature $(R=\infty)$, where the local refractive index is highest (see Fig.1e). This would erroneously predict light confinement in regions around $R=\infty$, rather than where $|R|$ is minimal [see Eq.(4)].

Using the confirmed JKC potential, we finally investigated the transport properties in the topological crystal by the application of a direct current (dc) force. Similarly to ordinary waveguide arrays, the dc force is expected to inhibit wave packet spreading and to cause an oscillatory motion of the wave packet [Bloch oscillations (BOs)] via the formation of a Wannier-Stark ladder spectrum [24], with the occurrence of Zener tunneling (ZT) at high dc forcing [26]. According to [27], a fictitious dc force is realized by fabricating the undulated slab slightly curved in the $z$-direction, which approximately preserves the $z$-invariance of the path $\gamma$, but imprints a transverse dc force in the $x$-direction. Our structure thus realizes a test bed for the observation of so-called topological BOs predicted in [21], where both effective lattice potential and dc force arise from the geometric deformation of a slab waveguide. The experimental observation of BOs in topological photonic crystals, corresponding to a broad input beam excitation, is shown in Fig. 4a, together with the numerical prediction (Fig. 4b). In order to visualize ZT, we doubled the transverse force by halving the longitudinal radius of curvature of the undulated slab waveguide. The results corresponding to broad beam excitation at the input plane are shown in Figs. 4k,d. Note that, as compared to Figs. 4a and 4b, ZT is now clearly visible because a considerable fraction of the wave packet does not experience Bragg reflection, but tunnels into higher propagation bands.

In conclusion, we experimentally observed a geometric potential for optical wave packets constrained on curved surfaces and showed the impact of the squeezing procedure on the resulting frictional potential. In particular, an optical version of a topological crystal [5] has been realized, in which the band structure of the crystal is determined by the geometric potential according to the JKC theory [3]. Our experiments shed new light onto the old and rather controversial problem of wave mechanics of quantum particles constrained on a curved 
space, and open the possibility to explore the interplay between topology and transport in low-dimensional curved structures. Our findings could also pave the way towards the investigation of photonic materials with topologically-controlled diffractive and refractive properties.

The authors acknowledge support by the Deutsche Forschungsgemeinschaft (Research Unit 532 and Leibniz program), the German Academy of Science Leopoldina (grant LPDS 2009-13), and the italian MIUR (PRIN 2008 project).

[1] B.S. DeWitt, Rev. Mod. Phys. 29, 377 (1957).

[2] H. Jensen and H. Koppe, Ann. Phys. (N.Y.) 63, 586 (1971).

[3] R.C.T. da Costa, Phys. Rev. A 23, 1982 (1981).

[4] M. Zheng, A. Jagota, E.D. Semke, B.A. Diner, R.S. Mclean, S.R. Lustig, R.E. Richardson, and N.G. Tassi, Nature Materials 2, 338 (2003).

[5] H. Aoki, M. Koshino, D. Takeda, and H. Morise, Phys. Rev. B 65, 035102 (2001).

[6] N.G. van Kampen and J.J. Lodder, Am. J. Phys. 52, 419 (1984).

[7] L. Kaplan, N.T. Maitra, and E.J. Heller, Phys. Rev. A 56, 2592 (1997).

[8] L.S. Schulman, Techniques and Applications of Path Integration, (Wiley, New York, 1981).

[9] M. Ikegami, Y. Nagaoka, S. Takagi, and T. Tanzawa, Prog. Theor. Phys. 88, 229 (1992).

[10] M.V. Entin and L.I. Magarill, Phys. Rev. B 64, 085330 (2001).

[11] J. Gravesen and M. Willatzen, Phys. Rev. A 72, 032108 (2005).

[12] E. Zhang, S. Zhang, and Q. Wang, Phys. Rev. B 75, 085308 (2007).

[13] G. Ferrari and G. Cuoghi, Phys. Rev. Lett. 100, 230403 (2008).

[14] H. Shima, H. Yoshioka, and J. Onoe, Phys. Rev. B 79, 201401(R) (2009).

[15] T. Schwartz, G. Bartal, S. Fishman, and M. Segev, Nature 446, 52 (2007).

[16] T. G. Philbin, C. Kuklewicz, S. Robertson, S. Hill, F. König, and U. Leonhardt, Science 319, 1367 (2008).

[17] A. Szameit, I.L. Garanovich, M. Heinrich, A.A. Sukhorukov, F. Dreisow, T. Pertsch, S. Nolte, A. Tünnermann, and Y.S. Kivshar, Nature Phys. 5, 271 (2009).

[18] D. A. Genov, S. Zhang, and X. Zhang, Nature Phys. 5, 687 (2009).

[19] S. Longhi, Laser \& Photon. Rev. 3, 243 (2009). 
[20] K. Itoh, W. Watanabe, S. Nolte, and C. Schaffer, MRS Bulletin 31, 620 (2006).

[21] S. Longhi, Opt. Lett. 32, 2647 (2007).

[22] A. Szameit, F. Dreisow, H. Hartung, S. Nolte, and A. Tünnermann, Appl. Phys. Lett. 90, $241113(2007)$.

[23] T. M. Benson and P. C. Kendall, Variational techniques including effective and weighted index methods, in Progress in Electromagnetic Research (EMW, Cambridge, Mass., 1995), Vol. 10, pp. 1-40.

[24] R. Morandotti, U. Peschel, J. S. Aitchison, H. S. Eisenberg, and Y. Silberberg, Phys. Rev. Lett. 83, 4756 (1999); T. Pertsch, P. Dannberg, W. Elflein, A. Bräuer, and F. Lederer, Phys. Rev. Lett. 83, 4752 (1999); D. N. Christodoulides, F. Lederer, and Y. Silberberg, Nature 424, 817 (2003).

[25] The effective index $n_{e}(x)$ is defined from the eigenvalue equation $\partial_{y}^{2} G(x, y)+$ $[n(x, y) / \lambda]^{2} G(x, y)=\left[n_{e}(x) / \lambda\right]^{2} G(x, y)$ taking $x$ as a parameter (see Eq.(2) in Ref. [23]).

[26] M. Ghulinyan, C. J. Oton, Z. Gaburro, L. Pavesi, C. Toninelli, and D. S. Wiersma, Phys. Rev. Lett. 94, 127401 (2005); H. Trompeter, T. Pertsch, F. Lederer, D. Michaelis, U. Streppel, A. Bräuer, and U. Peschel, Phys. Rev. Lett. 96, 023901 (2006).

[27] G. Lenz, I. Talanina, and C. M. de Sterke, Phys. Rev. Lett. 83, 963 (1999); N. Chiodo, G. Della Valle, R. Osellame, S. Longhi, G. Cerullo, R. Ramponi, P. Laporta, and U. Morgner, Opt. Lett. 31, 1651 (2006). 

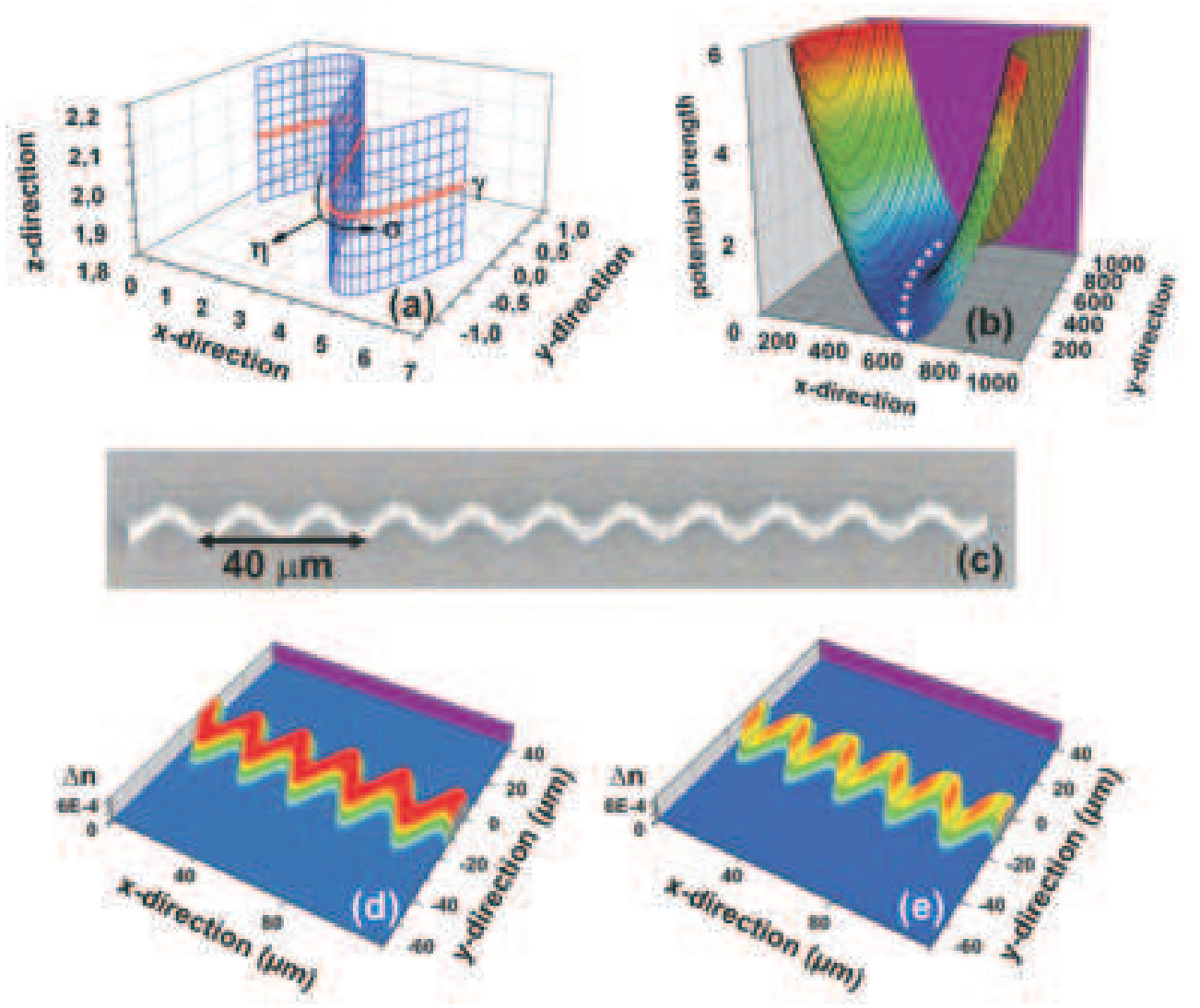

FIG. 1: (a) Sketch of a two-dimensional cylindrical (i.e. $z$-invariant) curved surface with the curvilinear coordinates $(\sigma, \eta)$. (b) The forced path of a wave packet in a constraining potential. (c) Microscope image of the front facet of the undulating guiding layer that realizes a one-dimensional topological crystal. (d) Homogeneous refractive index change yielding non-ideal squeezing. (e) Corrected refractive index distribution, resulting in ideal JKC conditions. 


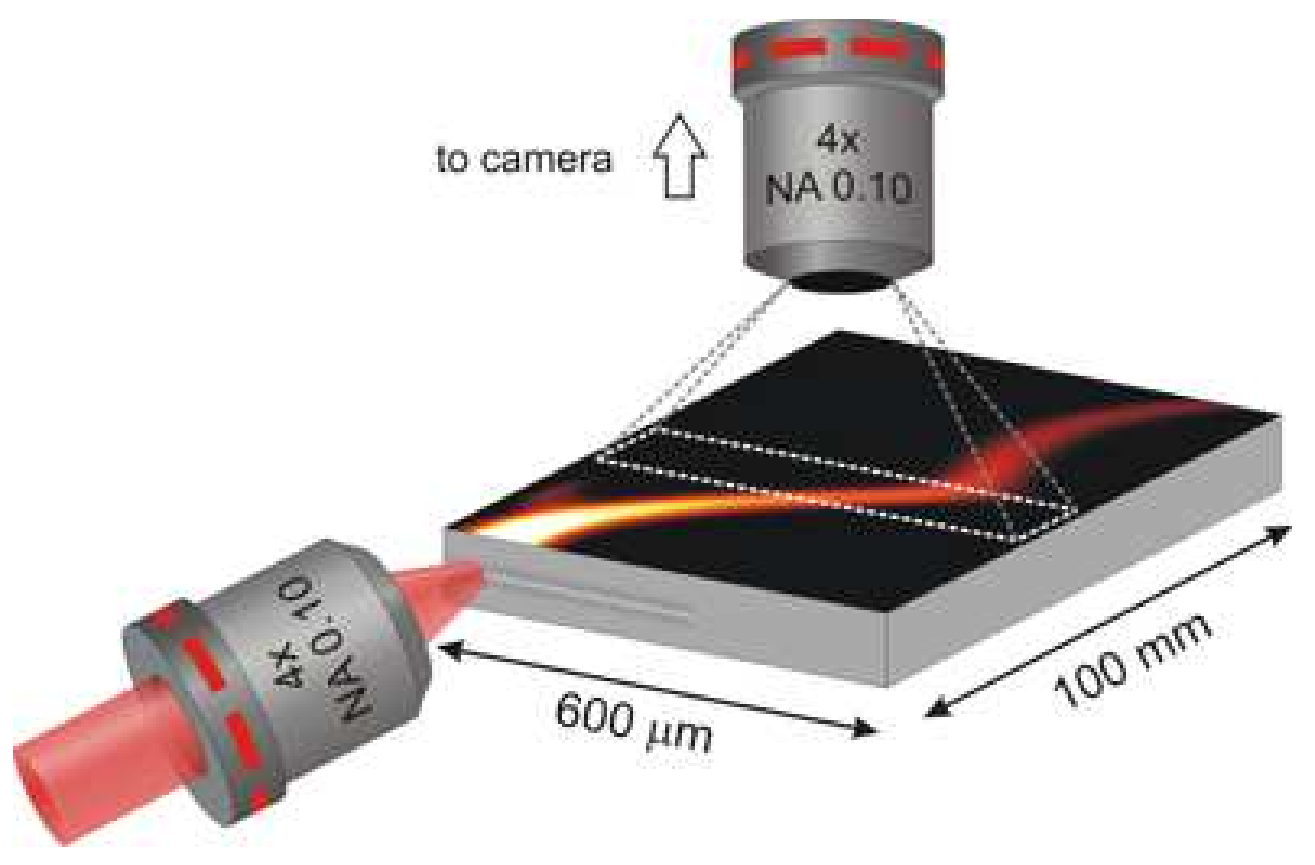

FIG. 2: Measurement setup using fluorescence microscopy. 

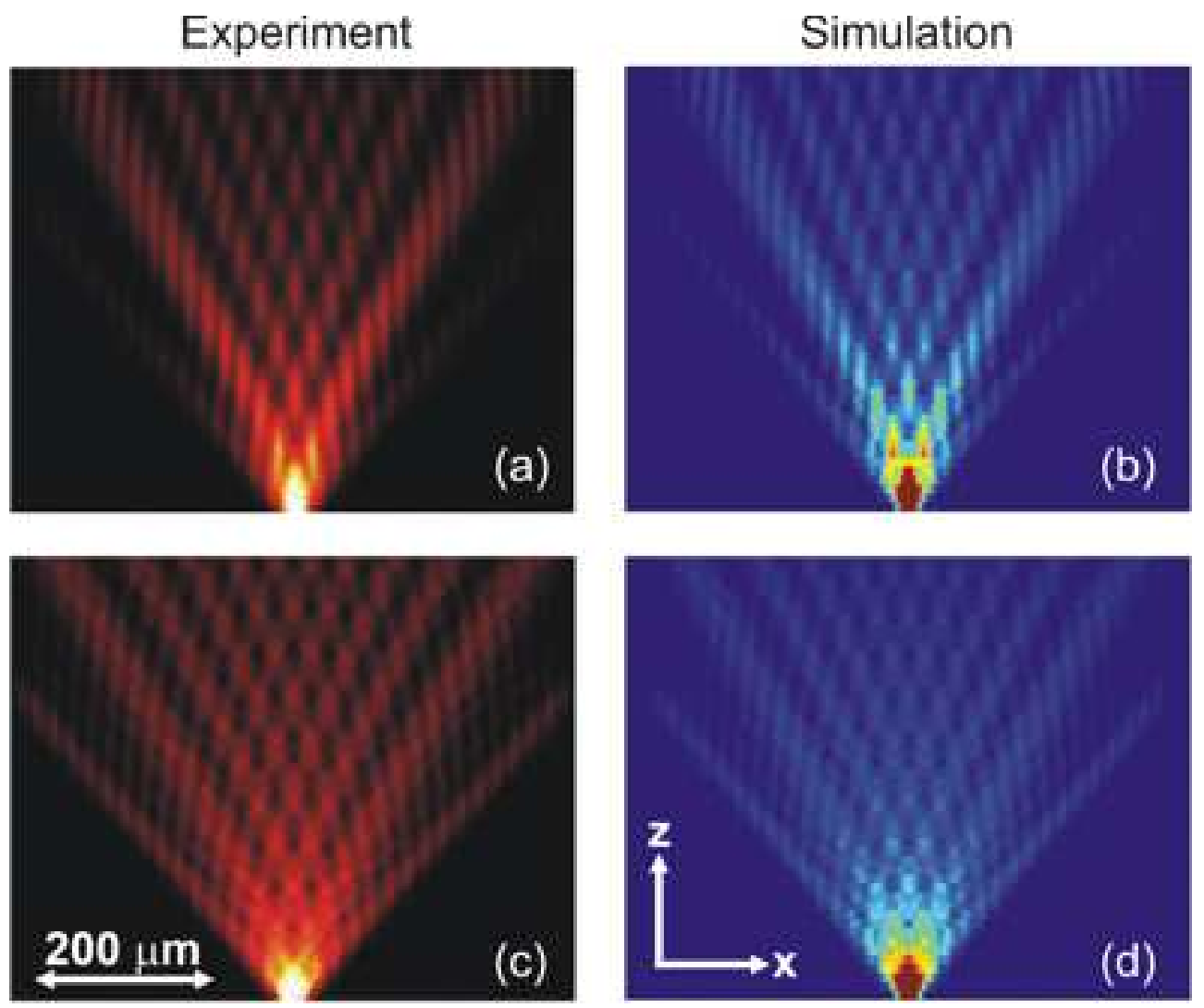

FIG. 3: (a) Measurement of the light propagation in a non-ideal squeezing potential, and (b) corresponding numerical confirmation. (c) Measurement of the light propagation under ideal JKC conditions, and (d) corresponding numerical confirmation. 


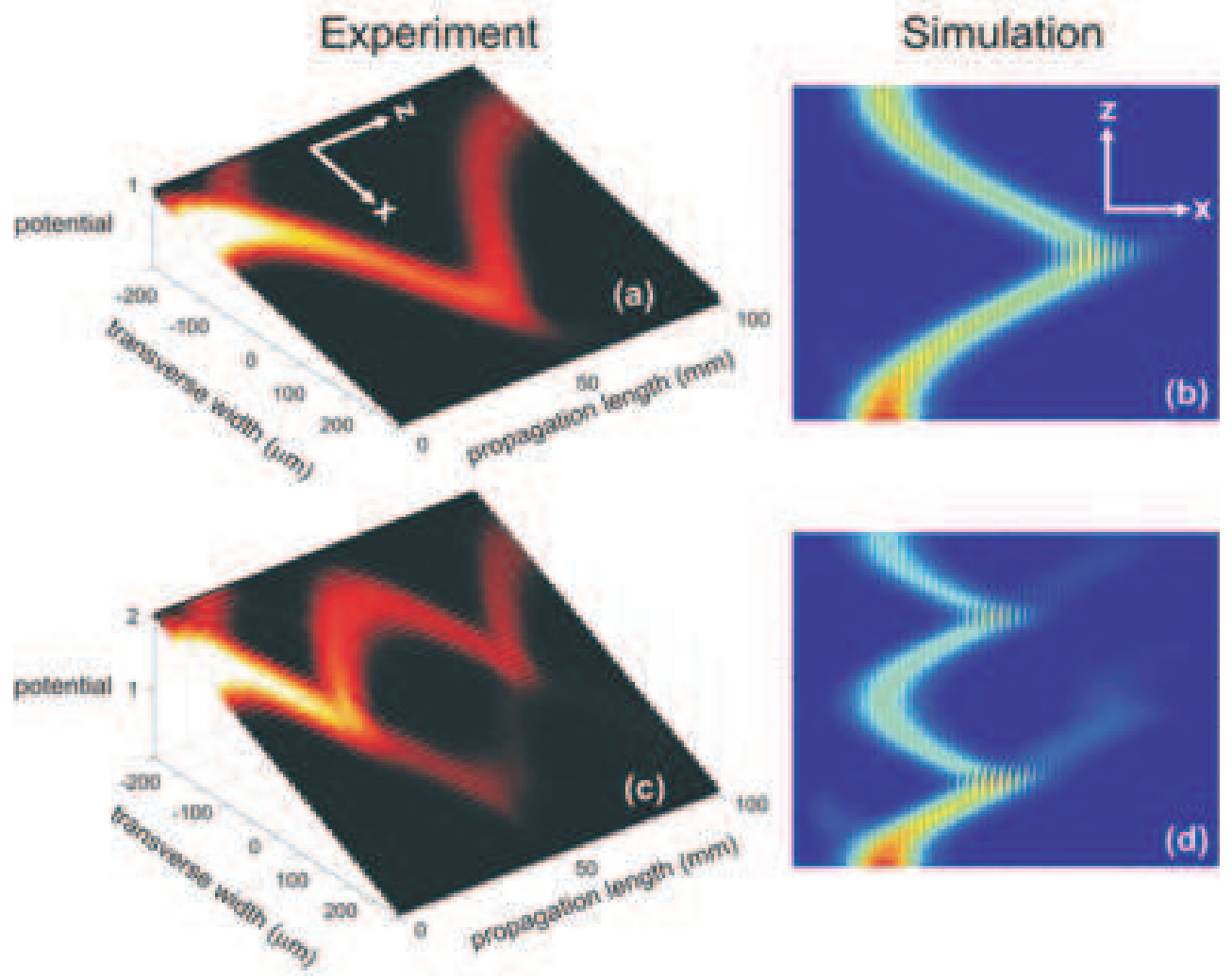

FIG. 4: (a) Dynamics of light in an optical topological crystal for broad beam excitation, when a transverse dc force is applied. (b) Numerical confirmation. (c) Zener tunneling for broad beam excitation in a high potential gradient. (d) Numerical confirmation. Note that the transverse dc force is applied by slightly curving the guiding layer in the $z$-direction. For reasons of visibility, this curvature has been numerically removed from the experimental images. Instead, the transverse gradient is sketched by tilting the images. 